findings, the difficulty in discerning which findings are of medical value, and the slow pace at which fuller knowledge of the links between genetics and disease is actually providing better diagnosis and treatment options.

On page 772 of this issue, Nature tells the story of Hugh Rienhoff, a trained geneticist and biotechnology entrepreneur, whose daughter was born with a collection of congenital defects. He has taken it upon himself to try to find out what the genetic cause might be - actually buying lab equipment and having her genes sequenced himself. $\mathrm{He}$ has even posted information about her condition, his theories as to what's causing it, and parts of her genetic sequence on the Internet.

Given the sharply falling costs of equipment and the wealth of information that is publicly available, we are getting to the point at which almost anyone with access to the Internet can do this. If that sounds a little scary, then perhaps it ought to. Scientists and patient advocates have always enjoyed a delicate relationship. Researchers are not prone to welcome what they may see as the intrusion of the public in the laboratory. And there is every chance that some people in Rienhoff's position will waste money pursuing dead ends. On the other hand, as more people begin to take an interest in rare or undiscovered

\section{Criteria creep}

\section{The politically motivated extension of a US stem- cell registry makes no scientific sense.}

$\Lambda$ steady lament from American biologists is that the human embryonic stem-cell lines that they can work on using federal research money are too old, and too few in number. But researchers will draw scant comfort from a White House executive order, issued on 20 June, that could sharply increase the number of cell lines on the Human Embryonic Stem Cell Registry - the list of cell lines that can be studied with support from the National Institutes of Health (NIH).

The executive order was issued by President Bush on the same day that he vetoed legislation that would have permitted funding to be used on research with additional embryonic stem-cell lines. It will replace the word 'embyronic' with 'pluripotent' in the registry's name, and thus add adult stem-cell lines to the registry - even though these are already eligible for federal funds. This political sleight of hand seems intended to increase the number of cell lines listed without adding new lines of embryonic cells.

For this to happen, senior NIH officials must now waste time trying to establish unarguable criteria that will affirm a cell line as pluripotent (see www.nature.com/stemcells). By the end of this month, they are expected to release an application form for researchers wishing to add new, non-embryonic lines to the list.

Pluripotency - which basically means that a cell can grow into any sort of body cell - is commonly evaluated in mouse cells by mixing candidate cells into mouse embryos and observing their subsequent development. But equivalent experiments cannot be ethically conducted with human cells, leaving no robust method for confirming their pluripotency. Indeed, pluripotency has yet to be formally proved disorders, more useful information is likely to be unearthed about both their genetics and their treatment.

But this means that clinical geneticists will have to revise the professional and ethical framework for collaborating with patients and their advocates, to help ensure that the information from the public provides clarity and not confusion. Some scientists are already thinking about how best to organize such information. On page 783, for example, Steven Brenner "Scientists and patient advocates have always enjoyed a delicate relationship."

of the University of California, Berkeley, proposes a 'genome commons' to aggregate the accumulated knowledge on human genetic and phenotypic diversity.

At the same time, members of the public who choose to embrace a do-it-yourself approach to science need to be aware that they should not abandon existing, rational treatment options. And they should know that the fruits of their labours will rarely include the cast-iron answers that they may be seeking. For, as is so often the case in science, the most likely result of their efforts will be yet more unanswered questions for others to probe.

in human cells in vitro - including in embryonic cells. There is also currently no way to prove that cells derived from embryonic and nonembryonic sources have equivalent capabilities to generate the specialized cells that could be useful in drug discovery and cell therapy.

The executive order calls for the thorough cataloguing of stem cells derived in what it calls "ethically responsible ways" - meaning, in the White House's parlance, that they are derived without creating, harming or destroying an embryo. The order further calls for the prioritization of new grants to study these lines. But no additional money is being allocated for this work, meaning that it can only proceed at the expense of other research supported by the NIH.

Flexible cells from non-embryonic sources do offer exciting possibilities: perhaps adult human cells can be reprogrammed, and cells from the testis and amniotic fluid can be coaxed into an array of functioning tissues. If such cells can be derived from individual patients with diseases, these non-embryonic sources could be of great value. But this value is more likely to be unleashed if they are studied alongside embryonic stem cells, rather than in their place.

On 9 August 2001, when Bush first announced his restriction of federal research funding to embryonic stem-cell lines already derived by that date, his officials suggested that researchers would be able to work with about 60 lines. But the true number has turned out to be about 20 , of which only a dozen are commonly used. NIH director Elias Zerhouni told a Senate committee back in March that the range of embryonic stem cells currently available to US researchers is insufficient, and is hampering scientific innovation and biomedical research.

The NIH has been obliged by law to come up with a plan for implementing the executive order. It will no doubt make the best of a difficult situation, and come up with some criteria for pluripotency. It is regrettable that one of the world's leading research agencies should be required to make avowedly scientific distinctions along lines drawn up to suit the administration's political requirements. 